\title{
Upper Bound in Change in Initial Value for “Time Component" of Pre Planckian Metric Tensor, for a Cosmological “Constant” Universe
}

\author{
Andrew W. Beckwith \\ Physics Department, Chongqing University, College of Physics, Chongqing University Huxi Campus, Chongqing, China \\ Email: Rwill9955b@gmail.com, abeckwith@uh.edu
}

How to cite this paper: Beckwith, A.W. (2017) Upper Bound in Change in Initial Value for "Time Component" of Pre Planckian Metric Tensor, for a Cosmological "Constant" Universe. Journal of High Energy Physics, Gravitation and Cosmology, 3, 657-662.

https://doi.org/10.4236/jhepgc.2017.34050

Received: June 2, 2017

Accepted: October 16, 2017

Published: October 19, 2017

Copyright $\odot 2017$ by author and Scientific Research Publishing Inc. This work is licensed under the Creative Commons Attribution International License (CC BY 4.0).

http://creativecommons.org/licenses/by/4.0/

\begin{abstract}
Beginning with Pebble's restatement of the Roberson-Walker line element, we obtain a way, afterwards, to calculate the relationship between an initial value of the "cosmological constant" and the value of fluctuations in the time component of the metric tensor $g$ (tot). We assume, in doing so that the value of the cosmological "constant" does not change from its initial formation. We close with speculations as to how this ties into other issues in the conclusion.
\end{abstract}

\section{Keywords}

Scale Factor, Initial Space-Time Volume, Modified HUP

\section{Basics, i.e. Roberson Walker Metric, Hubble Parameter, and Initial Density}

We start off with using the Roberson Walker metric, i.e. using [1] and in doing so, referencing page 74 of [1] we have that if we access using $R$ as the radius of a 3 sphere, in the Line metric, for the Roberson-Walker formulation, as well as a fixed volume of space, occupied cosmologically after a world line time, $t$, as

$$
V(3 \text {-sphere-volume })=2 \pi^{2}(a(t) \cdot R)^{3}
$$

Here, $a(t)$ is a scale factor, with the scale factor $=1$ in the present era, and being as low as 10 - 55 in Planck time regimes. If so then, if we speculate upon a density drop off, given phenomenologically by

$$
\rho(\text { Space-time-energy-density }) \sim \rho=\rho(\text { initial }) \cdot \exp (-\tilde{\tilde{\alpha}} t)
$$

And, then use of the evolution Equation (3), on the LHS, the time derivative of density, as given by 


$$
\dot{\rho}=-3 H \cdot\left(\rho+\left(P / c^{2}\right)\right)
$$

As well as looking at the generalized Chapyron Gas model for DM and DE [2] given as

$$
P=-A / \rho^{\omega} ; 0 \leq \omega \leq 1
$$

Then the density function for space time, as referenced in Equation (2) has an initial value of the form, if the volume is proportional to the cube of Planck length, $l_{P}^{3}$, then we will write, to first approximation, where $A=1 / 3$ by the radiation regime of space-time. Here we are assuming an invariant $\Lambda$ for the cosmological constant, with its value in early time the same as today, i.e. no Quintessence

$$
\rho \sim \frac{3}{\tilde{\tilde{\alpha}}} \cdot(1 \pm A) \cdot \Lambda+H . O . T
$$

The rest of this document will use a derivation by the author modified HUP [3] as to this Equation (5).

\section{Basic Background on the Heisenberg Uncertainty}

Principle, as Used by This Document

$$
\begin{aligned}
& (\Delta l)_{i j}=\frac{\delta g_{i j}}{g_{i j}} \cdot \frac{l}{2} \\
& (\Delta p)_{i j}=\Delta T_{i j} \cdot \delta t \cdot \Delta A
\end{aligned}
$$

If we use the following, from the Roberson-Walker metric [3] [4] [5] [6].

$$
\begin{aligned}
& g_{t t}=1 \\
& g_{r r}=\frac{-a^{2}(t)}{1-k \cdot r^{2}} \\
& g_{\theta \theta}=-a^{2}(t) \cdot r^{2} \\
& g_{\phi \phi}=-a^{2}(t) \cdot \sin ^{2} \theta \cdot d \phi^{2}
\end{aligned}
$$

Following Unruh [5] [6], write then, an uncertainty of metric tensor as, with the following inputs

$$
a^{2}(t) \sim 10^{-110}, r \equiv l_{P} \sim 10^{-35} \text { meters }
$$

Then, the surviving version of Equation (6) and Equation (7) is, then, if $\Delta T_{t t} \sim \Delta \rho$ [3] [4] [5] [6]

$$
\begin{aligned}
& V^{(4)}=\delta t \cdot \Delta A \cdot r \\
& \delta g_{t t} \cdot \Delta T_{t t} \cdot \delta t \cdot \Delta A \cdot \frac{r}{2} \geq \frac{\hbar}{2} \\
& \Leftrightarrow \delta g_{t t} \cdot \Delta T_{t t} \geq \frac{\hbar}{V^{(4)}}
\end{aligned}
$$

Equation (9) is such that we can extract, up to a point the HUP principle for uncertainty in time and energy, with one very large caveat added, namely if we use the fluid approximation of space-time [7] 


$$
T_{i i}=\operatorname{diag}(\rho,-p,-p,-p)
$$

Then by [3]

$$
\Delta T_{t t} \sim \Delta \rho \sim \frac{\Delta E}{V^{(3)}}
$$

Then, by [3]

$$
\begin{aligned}
& \delta t \Delta E \geq \frac{\hbar}{\delta g_{t t}} \neq \frac{\hbar}{2} \\
& \text { Unless } \delta g_{t t} \sim O(1)
\end{aligned}
$$

\section{Estimating of the $\Delta g_{t t}$ Term in Equation (12), as the Conclusion, with Consequences}

The summary of what we obtain here, is if

$$
\begin{aligned}
& \rho \sim \frac{3}{\tilde{\tilde{\alpha}}} \cdot(1 \pm A) \cdot \Lambda+H . O \cdot T \sim \frac{\Delta E}{l_{p}^{3}} \\
& \& A=1 / 3(\text { radiation }) \\
& \Leftrightarrow \Delta g_{t t} \sim \frac{\hbar \tilde{\tilde{\alpha}}}{\left(t_{\min } \sim \text { Planck-time }\right)} \cdot l_{p}^{3} \cdot(1 \pm A) \cdot \Lambda_{\text {Today's-value }}
\end{aligned}
$$

For our purposes, this corresponds to having $\tilde{\tilde{\alpha}}$ fairly large but not infinite, but also the decisive factor in the reduction of energy density as given in Equation (2), i.e. that even in the Pre Planckian regime, that we position the energy density for a dramatic drop in value. We do this preparation for a reduction in the energy density so that the value of $\Delta g_{t t}$ is very small and consistent with [8]. And also, what we are referring to as a phase shift, as for a change of state in the HUP, as delineated below:

$$
\begin{aligned}
& \delta t \Delta E \geq\left.\frac{\hbar}{\delta g_{t t}}\right|_{\text {Pre-Octonionic }} \underset{\text { change in phase, given byp phase } \delta_{0}}{\longrightarrow} \delta t \Delta E \geq\left.\hbar\right|_{\text {Octonionic }} \\
& \text { with } \delta t \geq \frac{\hbar}{\delta g_{t t} \Delta E} \text { FIXED }
\end{aligned}
$$

This matter of Octonionic and Pre Octonionic is being pursued separately by the author, but the notice of a phase shift, is in work which is consistent with work which Dr. Li and Dr. Yang did, in [9] and the reader can access Octonionic and Pre Octonionic states by the following:

Keep in mind one basic fact. If we restrict ourselves solely to Octonionic geometry, we are embedded deeply in only what the Standard Model of physics allows. We should though understand what is implied by the physics of the Octonionic structure and so the rest of this first discussion is devoted to it.

In [10], Wilson gives a generalized structure as to Octonionic geometry, and it is a generalized way to introduce higher level geometry into the formation of standard model physics. Crowell, in [11] examines its applications as to pre- 
sumed space-time structure. Also note what is said in [12] the take away from it, is that as quoted from [12], that there exists

Quote:

(A linkage to the) mathematics of the division algebras and the Standard Model of quarks and leptons with $\mathrm{U}(1) \times \mathrm{SU}(2) \times \mathrm{SU}(3)$ gauge fields.

End of quote:

Once again, if we have only $\mathrm{U}(1) \times \mathrm{SU}(2) \times \mathrm{SU}(3)$ gauge fields, we have only the standard model, and that if we wish to have a minimum time step, we need to go beyond the standard model.

The division algebras are linked to Octonionic structure in a way which is touched upon by Crowell [11], but the main take away is that in the Pre-Planckian space-time regime, that there was specific inputs which may explain some of the findings of [13] from a different perspective. As well as [14], it is worth reading, i.e. the reader, [15] as well as Reference [16] for Baez's summary as to the properties of Octonions as well. We also claim that resolution of these details will be important in falsification of the argument given in [17] as well. The uncertain principle as utilized can be checked against the generalized HUP as given in [18], and if or not a phase shift in early universe signals, GW, or what have you as given in [19] occurs, may give insight as to if there are extra dimensions as alleged in [20].

It is worth reviewing if this construction meets the experimental gravitational tests mentioned in [21] by Abbot et al. of LIGO which started experimental gravitational astronomy. Furthermore, the considerations of Octonionic space-time theory should be checked against the experimental tests mentioned by Corda, in [22] which could confirm or falsify different gravitational theoretical models. In addition, this can be further refined by the $2^{\text {nd }}$ Abbot paper, [23] which did a different mass range of black hole binary, as of [21].

In addition, all these can be used to also vet if [24], by Penrose, i.e. cyclic conformal cosmology, as written, is ruled out or confirmed by various experimental and modeling tests.

\section{Acknowledgements}

This work is supported in part by National Nature Science Foundation of China grant No. 11375279.

\section{References}

[1] Peebles, P.J.E.R. (1993) Principles of Physical Cosmology, Princeton Series in Physics. Princeton University Press, Princeton, NJ.

[2] Benaoum, H.B. (2012) Modified Chaplygin Gas Cosmology. Advances in High Energy Physics, 2012, Article ID: 357802. http://arxiv.org/abs/1211.3518 https://doi.org/10.1155/2012/357802

[3] Beckwith, A. (2016) Gedanken Experiment for Refining the Unruh Metric Tensor Uncertainty Principle via Schwartz Shield Geometry and Planckian Space-Time with Initial Nonzero Entropy and Applying the Riemannian-Penrose Inequality and 
Initial Kinetic Energy for a Lower Bound to Graviton Mass (Massive Gravity). Journal of High Energy Physics, Gravitation and Cosmology, 2, 106-124. https://doi.org/10.4236/jhepgc.2016.21012

[4] Gorbunov, D. and Rubakov, V. (2011) Introduction to the Theory of the Early Universe, Cosmological Perturbations and Inflationary Theory. World Scientific Publishing Pte. Ltd, Singapore.

[5] Unruh, W.G. (1986) Why Study Quantum Theory? Canadian Journal of Physics, 64, 128-130. https://doi.org/10.1139/p86-019

[6] Beckwith, A.W. (2016) Gedanken Experiment for Refining the Unruh Metric Tensor Uncertainty Principle via Schwarzschild Geometry and Planckian Space-Time with Initial Nonzero Entropy and Applying the Riemannian-Penrose Inequality and Initial Kinetic Energy for a Lower Bound to Graviton Mass (Massive Gravity). Journal of High Energy Physics, Gravitation and Cosmology, 2, 106-124. http://dx.doi.org/10.4236/jhepgc.2016.21012

[7] Kolb, E., and Turner, S. (1991) The Early Universe. Westview Press, Chicago, IL.

[8] Giovanni, M. (2008) A Primer on the Physics of the Cosmic Microwave Background. World Press Scientific, Hackensack, NJ. https://doi.org/10.1142/6730

[9] Li, F., and Yang, N. (2009) Phase and Polarization State of High Frequency Gravitational waves. Chinese Physics Letters, 236, 1-4.

[10] Wilson, R. Octonions. (2008)

http://www.maths.qmul.ac.uk/ raw/talks_files/octonions.pdf

[11] Crowell, L. (2005) Quantum Fluctuations of Space-Time World Press Scientific. World Scientific Series in Contemporary Chemical Physics, Vol. 25, Singapore.

[12] Dixon, G.M. (1994) Division Algebras: Octonions Quaternions Complex Numbers and the Algebraic Design of Physics. Mathematics and Its Applications, Kluwer Academic Publishers, London. https://doi.org/10.1007/978-1-4757-2315-1

[13] Beckwith, A. (2016) Gedanken Experiment Examining How Kinetic Energy Would Dominate Potential Energy, in Pre-Planckian Space-Time Physics, and Allow Us to Avoid the BICEP 2 Mistake. Journal of High Energy Physics, Gravitation and Cosmology, 2, 75-82. https://doi.org/10.4236/jhepgc.2016.21008

[14] Beckwith, A. (2016) Open Question: Could a Causal Discontinuity Explain Fluctuations in the CMBR Radiation Spectrum? Journal of High Energy Physics, Gravitation and Cosmology, 2, 186-208. https://doi.org/10.4236/jhepgc.2016.22018

[15] Baez, J. (2002) The Octonians. Bulletin of the American Mathematical Society, 39, 145-205. http://arxiv.org/abs/math/0105155v4 https://doi.org/10.1090/S0273-0979-01-00934-X

[16] Martel, H., Shapiro, P. and Weinberg, S. (1998) Likely Values of the Cosmological Constant. The Astrophysical Journal, 492, 29-40. http://iopscience.iop.org/article/10.1086/305016/fulltext/35789.text.html

[17] Easther, R. and Lowe, D. (1999) Holography, Cosmology and the Second Law of Thermodynamics. Physical Review Letters, 82, 4967-4970.

http://arxiv.org/abs/hep-th/9902088 https://doi.org/10.1103/PhysRevLett.82.4967

[18] Miao, Y.G. and Zhao, Y.J. (2013) Interpretation of the Cosmological Constant Problem within the Framework of Generalized Uncertainty Principle. http://arxiv.org/abs/1312.4118

[19] Abbott, B.P., et al. (2009) An Upper Limit on the Stochastic Gravitational-Wave Background of Cosmological Origin, Data. Nature, 460, 990. 
http://www.phys.ufl.edu/ tanner/PDFS/Abbott09Nature-Stochastic.pdf

[20] Clarkson, C. and Seahra, S. (2007) A Gravitational Wave Window on Extra Dimensions. Classical and Quantum Gravity, 24, F33. https://doi.org/10.1088/0264-9381/24/9/F01

[21] Abbott, B.P., et al. (2016) Observation of Gravitational Waves from a Binary Black Hole Merger. PRL, 116, Article ID: 061102.

https://physics.aps.org/featured-article-pdf/10.1103/PhysRevLett.116.061102

[22] Corda, C. (2009) Interferometric Detection of Gravitational Waves: The Definitive Test for General Relativity. International Journal of Modern Physics D, 18, 2275-2282. https://doi.org/10.1142/S0218271809015904

[23] Abbot, B.P., et al. (2016) GW151226: Observation of Gravitational Waves from a 22-Solar-Mass Binary Black Hole Coalescence. Physical Review Letters, 116, Article ID: 241103. https://doi.org/10.1103/PhysRevLett.116.241103

[24] Penrose, R. (2011) Cycles of Time. Alfred Knopf, New York. 